Journal of

Synchrotron

Radiation

ISSN 1600-5775

\title{
Characterization of spatial coherence of synchrotron radiation with non-redundant arrays of apertures
}

\author{
P. Skopintsev, A. Singer, J. Bach, L. Müller, B. Beyersdorff, S. Schleitzer, O. \\ Gorobtsov, A. Shabalin, R. P. Kurta, D. Dzhigaev, O. M. Yefanov, L. Glaser, \\ A. Sakdinawat, G. Grübel, R. Frömter, H. P. Oepen, J. Viefhaus and I. A. \\ Vartanyants
}

J. Synchrotron Rad. (2014). 21, 722-728

Copyright (C) International Union of Crystallography

Author(s) of this paper may load this reprint on their own web site or institutional repository provided that this cover page is retained. Republication of this article or its storage in electronic databases other than as specified above is not permitted without prior permission in writing from the IUCr.

For further information see http://journals.iucr.org/services/authorrights.html

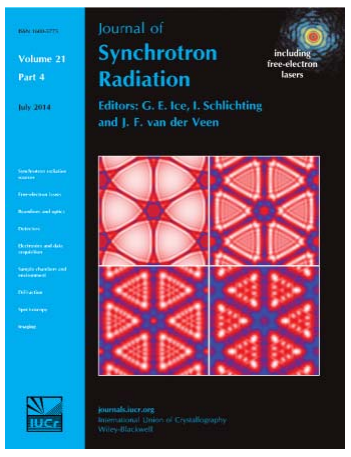

Synchrotron radiation research is rapidly expanding with many new sources of radiation being created globally. Synchrotron radiation plays a leading role in pure science and in emerging technologies. The Journal of Synchrotron Radiation provides comprehensive coverage of the entire field of synchrotron radiation research including instrumentation, theory, computing and scientific applications in areas such as biology, nanoscience and materials science. Rapid publication ensures an up-to-date information resource for scientists and engineers in the field.

Crystallography Journals Online is available from journals.iucr.org 
Journal of

Synchrotron

ISSN 1600-5775

Accepted 27 March 2014
Radiation

Received 24 February 2014

\section{Characterization of spatial coherence of synchrotron radiation with non-redundant arrays of apertures}

\author{
P. Skopintsev, ${ }^{a, b, c}$ A. Singer, ${ }^{a} \ddagger$ J. Bach, ${ }^{d}$ L. Müller, ${ }^{a}$ B. Beyersdorff, ${ }^{d}$ S. Schleitzer, ${ }^{a}$ \\ O. Gorobtsov, ${ }^{a, b}$ A. Shabalin, ${ }^{a}$ R. P. Kurta, ${ }^{a}$ D. Dzhigaev, ${ }^{a, e}$ O. M. Yefanov, ${ }^{a}$ \\ L. Glaser, ${ }^{a}$ A. Sakdinawat, ${ }^{f}$ G. Grübel, ${ }^{a, g}$ R. Frömter, ${ }^{d}$ H. P. Oepen, ${ }^{d}$ J. Viefhaus ${ }^{a}$ \\ and I. A. Vartanyants $\mathrm{s}^{\mathrm{a}, \mathrm{e}}$ *

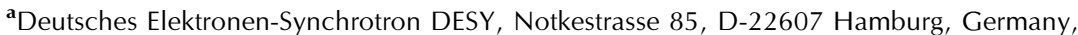 \\ b National Research Center 'Kurchatov Institute', Kurchatov Square 1, 123182 Moscow, Russia, \\ 'Moscow Institute of Physics and Technology (State University), Dolgoprudny, Moscow Region \\ 141700, Russia, 'Universität Hamburg, Institut für Angewandte Physik, D-20355 Hamburg, \\ Germany, 'National Research Nuclear University, 'MEPhl', 115409 Moscow, Russia, 'SLAC \\ National Accelerator Laboratory, 2575 Sand Hill Road, Menlo Park, CA 94025-7015, \\ USA, and ${ }^{\mathrm{g}}$ The Hamburg Center for Ultrafast Imaging, D-22761 Hamburg, Germany. \\ *E-mail: ivan.vartaniants@desy.de
}

\begin{abstract}
A method to characterize the spatial coherence of soft X-ray radiation from a single diffraction pattern is presented. The technique is based on scattering from non-redundant arrays (NRAs) of slits and records the degree of spatial coherence at several relative separations from 1 to $15 \mu \mathrm{m}$, simultaneously. Using NRAs the spatial coherence of the X-ray beam at the XUV X-ray beamline P04 of the PETRA III synchrotron storage ring was measured as a function of different beam parameters. To verify the results obtained with the NRAs, additional Young's double-pinhole experiments were conducted and showed good agreement.
\end{abstract}

(C) 2014 International Union of Crystallography

Keywords: coherence; interference; synchrotron X-ray sources.

\section{Introduction}

It is well established that the present-day third-generation synchrotrons are partially coherent sources (Winick, 1980; Attwood, 1999; Vartanyants \& Singer, 2010). With the construction of these facilities, new research areas utilizing partial coherence of X-ray radiation have emerged. The most prominent experimental techniques are coherent X-ray diffractive imaging (CXDI) (Vartanyants et al., 2010; Chapman \& Nugent, 2010; Mancuso et al., 2010), X-ray holography (Eisebitt et al., 2004; Stickler et al., 2010) and $\mathrm{X}$-ray photon correlation spectroscopy (XPCS) (Grübel \& Zontone, 2004). In CXDI, static real-space images of the sample are obtained by phase-retrieval techniques (Fienup, 1982), while correlation techniques are applied in XPCS to explore system dynamics (Goodman, 2007). The key feature of the methods utilizing the high degree of coherence is the interference of the field scattered by different parts of the sample under study. Hence, spatial coherence across the sample is essential and understanding the coherence proper-

$\ddagger$ Present address: University of California, San Diego, CA 92093, USA. ties of the beams at new-generation X-ray sources is of crucial importance for the scientific community. Moreover, a detailed knowledge of the beam coherence can be used to improve the resolution obtained in the CXDI phase retrieval (Whitehead et al., 2009).

The Young's double-pinhole experiment is the most direct technique to map out the spatial coherence. The method has been successfully applied for soft X-ray synchrotron radiation (Chang et al., 2000; Paterson et al., 2001) and, recently, for free-electron lasers (FELs) (Singer et al., 2008, 2012; Vartanyants et al., 2011). However, a single double-pinhole experiment yields the spatial coherence at only one relative distance, the distance between the pinholes. To fully characterize the spatial coherence, several measurements at different pinhole separations are required. Other techniques have been implemented to measure the spatial coherence, such as X-ray grating interferometry (Pfeiffer et al., 2005), phase-space tomography (Raymer et al., 1994; Tran et al., 2007), methods which utilize scattering on Brownian particles (Alaimo et al., 2009), and measurements of the intensity correlations (Gluskin et al., 1999; Yabashi et al., 2001; Singer et al., 2013). Similar to the double-pinhole experiment, the 
aforementioned techniques require a number of measurements to fully determine the spatial coherence properties of the probing radiation.

Here we present a method to completely characterize the spatial coherence function of the soft X-ray radiation from a single diffraction pattern. This method utilizes a non-redundant array (NRA), i.e. a mask with multiple slits where each slit separation appears only once. Each pair of slits acts as a single Young's interferometer, and a measurement using an NRA can be considered as a series of double-pinhole interferograms acquired simultaneously. The signal from different slit pairs can be discriminated due to the uniqueness of each slit separation. This is in contrast to coherence measurements using a uniformly redundant array (URA), where each slit separation appears more than once (Lin et al., 2003). The analysis of URA measurements is quite sensitive to the wavefront of the incident radiation (Vartanyants et al., 2010), a limitation that is not present in NRA measurements. NRAs were previously used to measure spatial coherence of a $\mathrm{He}-\mathrm{Ne}$ laser (Mejía \& González, 2007; González \& Mejía, 2011).

In this paper we demonstrate spatial coherence measurements performed at the XUV X-ray beamline P04 at PETRA III using NRA apertures.

\section{Theory}

In the theory of optical coherence, the statistical properties of the radiation, including spatial coherence, are described by correlation functions of the electric field. The mutual coherence function (MCF) is defined as (Goodman, 1985)

$$
\Gamma\left(\mathbf{r}_{1}, \mathbf{r}_{2} ; \tau\right)=\left\langle E^{*}\left(\mathbf{r}_{1}, t\right) E\left(\mathbf{r}_{2}, t+\tau\right)\right\rangle,
$$

where $E\left(\mathbf{r}_{1}, t\right)$ and $E\left(\mathbf{r}_{2}, t+\tau\right)$ are the field values at the positions $\mathbf{r}_{1}$ and $\mathbf{r}_{2}$ and times $t$ and $t+\tau$, and $\langle\cdots\rangle$ is the ensemble average. The intensity $I(\mathbf{r})$ and complex degree of coherence $\gamma_{12}(\tau)$ can be readily obtained from the MCF,

$$
I(\mathbf{r})=\Gamma(\mathbf{r}, \mathbf{r} ; 0), \quad \gamma_{12}(\tau)=\frac{\Gamma\left(\mathbf{r}_{1}, \mathbf{r}_{2} ; \tau\right)}{\left[I\left(\mathbf{r}_{1}\right) I\left(\mathbf{r}_{2}\right)\right]^{1 / 2}} .
$$

If the time delay $\tau$ is much smaller than the coherence time $\tau_{\mathrm{c}}$, $\gamma_{12}(\tau)$ can be well approximated by the complex coherence factor (CCF) $\gamma_{12}=\gamma_{12}(0)$ (Goodman, 1985) that does not depend on the time delay $\tau$.

In the frame of the Gaussian Schell-model, which in most cases provides sufficient description of synchrotron radiation (Vartanyants \& Singer, 2010), the intensity profile and the $\mathrm{CCF}$ are both Gaussian functions. The beam size in this model is characterized by its root mean square (r.m.s.) width $\sigma$ of the intensity and the transverse coherence length $l_{\mathrm{c}}$ can be defined as the r.m.s. width of $\left|\gamma_{12}\right|$. To characterize the transverse coherence by one number, the global degree of coherence can be introduced (Saldin et al., 2008; Vartanyants \& Singer, 2010),

$$
\zeta=\left(l_{\mathrm{c}} / \sigma\right)\left[4+\left(l_{\mathrm{c}} / \sigma\right)^{2}\right]^{-1 / 2} .
$$

To measure the CCF of the radiation an arrangement of apertures can be used as a scattering object. The correlations between the field scattered at the apertures and propagated to the detection plane can be recorded as intensity modulations or interference fringes. For the narrow bandwidth radiation, the far-field diffraction pattern $I(\mathbf{q})$ of $N$ identical apertures can be expressed in the following way (Mejía \& González, 2007; González \& Mejía, 2011),

$$
I(\mathbf{q})=I_{\mathrm{S}}(\mathbf{q})\left\{C_{0}+\sum_{i \neq j}^{N} C_{i j} \exp \left[i\left(\mathbf{q} \cdot \mathbf{d}_{i j}+\alpha_{i j}\right)\right]\right\},
$$

where coefficients $C_{0}$ and $C_{i j}$ are defined as

$$
C_{0}=\sum_{i} I_{i}, \quad C_{i j}=\left|\gamma_{i j}\right|\left(I_{i} I_{j}\right)^{1 / 2} .
$$

Here $I_{i}$ is the intensity incident on the aperture $i,{ }^{\mathbf{1}}\left|\gamma_{i j}\right|$ is the modulus of the CCF at the aperture separation vector $\mathbf{d}_{i j}=-\mathbf{d}_{j i}, \alpha_{i j}=-\alpha_{j i}$ is the relative phase, $I_{\mathrm{S}}(\mathbf{q})$ is the intensity distribution on the detector due to beam diffraction on a single aperture, and $\mathbf{q}$ is the momentum transfer vector. If the aperture has the form of a pinhole, then $I_{S}(\mathbf{q})$ is the well known Airy pattern. For a rectangular slit, $I_{S}(\mathbf{q})$ has the shape of a squared sinc function. An example of the intensity distribution $I(\mathbf{q})$ for diffraction on six and two slits is shown in Figs. 1(a) and 1(c).

The interference pattern in equation (4) can be conveniently analyzed using the Fourier transform $\hat{I}(\Delta \mathbf{x})$ of $I(\mathbf{q})$, which for $N$ apertures is given by (Bartels et al., 2002; Mejía \& González, 2007; González \& Mejía, 2011)

$$
\begin{aligned}
\hat{I}(\Delta \mathbf{x})= & \hat{I}_{\mathrm{S}}(\Delta \mathbf{x}) \otimes\left\{C_{0} \delta(\Delta \mathbf{x})\right. \\
& \left.+\sum_{i \neq j}^{N} C_{i j}\left[\exp \left(i \alpha_{i j}\right) \delta\left(\Delta \mathbf{x}-\mathbf{d}_{i j}\right)\right]\right\},
\end{aligned}
$$

where $\delta(\Delta \mathbf{x})$ is the Dirac delta function, $\hat{I}_{\mathrm{S}}(\Delta \mathbf{x})$ is the Fourier transform of $I_{\mathrm{S}}(\mathbf{q})$, the symbol $\otimes$ denotes the convolution, and $\Delta \mathbf{x}$ denotes the relative distance. It is clear from expression (6) that the peak maximum at each peak separation $\mathbf{d}_{i j}$ is given by coefficients $C_{i j}$. If the intensities $I_{i}$ incident on the apertures are known, the CCF can be readily obtained from equation (5) as

$$
\left|\gamma_{i j}\right|=C_{i j} /\left(I_{i} I_{j}\right)^{1 / 2}
$$

for all $\mathbf{d}_{i j}$. An NRA with $N$ slits shows $N(N-1)+1$ individual peaks in the Fourier transform $\hat{I}(\Delta \mathbf{x})$ of the measured intensity $I(\mathbf{q})$, whereas each peak corresponds to interference between a unique combination of two slits. According to equation (7), each peak in equation (6) corresponds to a unique value of $\left|\gamma_{i j}\right|$. Typical distributions $\hat{I}(\Delta \mathbf{x})$ with 31 and 3 peaks, corresponding to six and two slit apertures, are shown in Figs. $1(b)$ and $1(d)$.

It is clear that the outcome of the measurement strongly depends on the design of the NRA. To optimize the NRA

\footnotetext{
$\overline{\mathbf{1}}$ Here we assume that the incident intensity is constant across a single aperture.
} 

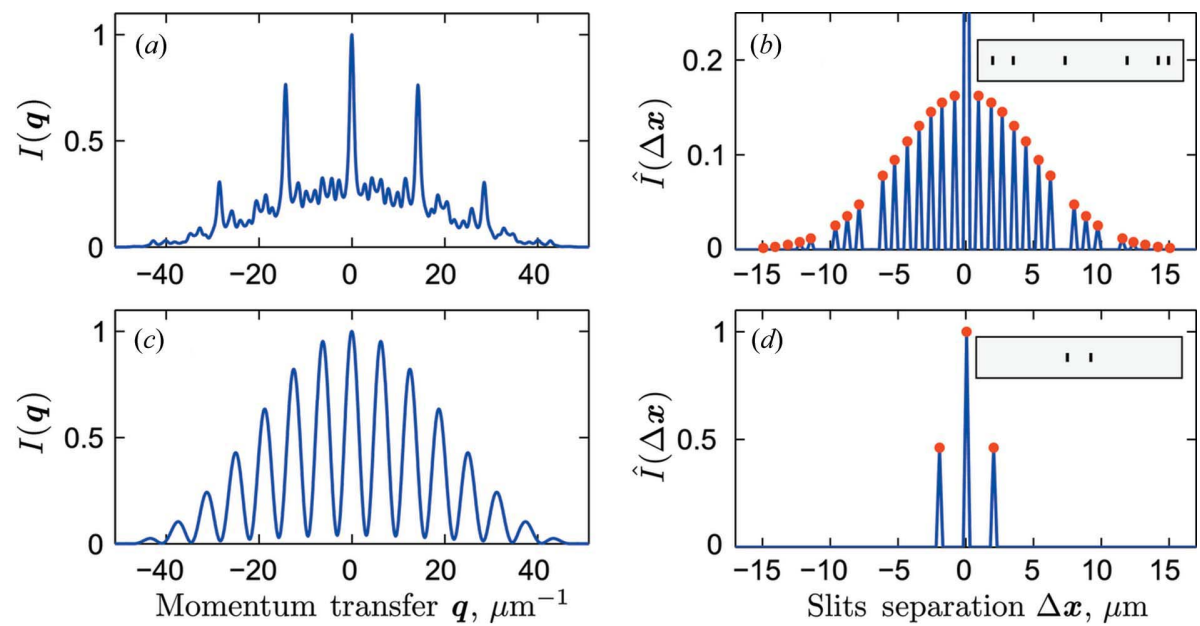

Figure 1

$(a, c)$ Simulated diffraction patterns $I(q)$ from NRAs of six $(a)$ and two $(c)$ slits. $(b, d)$ Fourier transform $\hat{I}(\Delta x)$ of corresponding diffraction patterns from six $(b)$ and two $(d)$ slits. Red points in $(b, d)$ denote the central and satellite peaks' maxima $C_{0}$ and $C_{i j}$, respectively. The insets in $(b)$ and $(d)$ show the slits' relative positions used in simulations. The radiation was uniformly distributed in the slits and had a coherence length of $l_{\mathrm{c}}=5 \mu \mathrm{m}$.

structure one possibility would be to find a uniform spatial distribution of the peaks. It can be shown that such an ideal NRA with a uniform distribution of the peaks can only be achieved with four slits (Dollas et al., 1998). For more than four slits the peaks can be uniformly distributed, but some of them will be missing. However, it is still possible to find an arrangement of $N$ slits with as few missing peaks as possible. In this work we designed NRAs using a so-called Golomb ruler ${ }^{2}$ that has integer marks with a distinct distance between every two marks.

\section{Experiment}

The coherence measurements were performed at the Variable Polarization XUV beamline P04 (Viefhaus et al., 2013) of the PETRA III synchrotron radiation source at DESY in Hamburg during commissioning. The beamline set-up is schematically presented in Fig. 2. A $5 \mathrm{~m}$ APPLE-II type

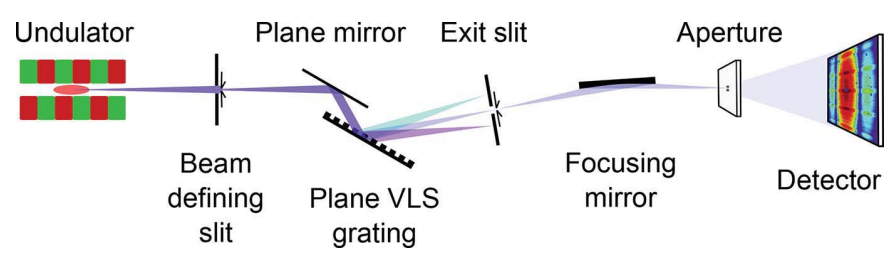

Figure 2

Experimental set-up. Synchrotron radiation is generated by a $5 \mathrm{~m}$ APPLE-II type undulator and is transmitted through the beamline including a beam-defining slit, monochromator comprised of a VLS plane grating and pair of plane mirrors, exit slit, focusing mirror and collimating mirror. The beam is focused on the apertures by the focusing mirror and forms a diffraction pattern on the CCD detector positioned in the farfield. The horizontal plane mirror and the collimating mirror are not shown in the figure.

\footnotetext{
${ }^{2}$ For a list of all currently available optimal Golomb rulers, see Lam \& Sarwate (1988) (see also Wikipedia, 2014).
}

helical undulator with 72 magnetic periods was tuned to deliver $400 \mathrm{eV}$ (wavelength $\lambda=3.1 \mathrm{~nm}$ ) photons. The beam further propagated to the end-station through several optical elements, including a beam-defining slit (27 $\mathrm{m}$ downstream from the undulator), horizontal plane mirror $(35 \mathrm{~m})$ and vertical plane mirror together with a plane varied-line-spacing (VLS) grating $(46 \mathrm{~m})$. The VLS grating focused the beam at the exit slit $(71 \mathrm{~m})$. An elliptical mirror $(78.5 \mathrm{~m})$ focused the beam vertically to the sample position $(81 \mathrm{~m})$. Horizontally the beam was collimated by a cylindrical mirror $(79.1 \mathrm{~m})$ and defined by a slit in front of the sample. All mirrors were designed to accept $6 \sigma$ r.m.s. of the beam size.

NRAs and double pinholes were manufactured in an opaque screen [see Vartanyants et al. (2011) for a detailed description of the manufacturing process] and were used to map out the transverse coherence of the beam. The distance between individual slits in the NRA aperture was varying between $1 \mu \mathrm{m}$ and $15 \mu \mathrm{m}$. The slits were positioned at $-7.5 \mu \mathrm{m},-6.62 \mu \mathrm{m},-3.97 \mu \mathrm{m}, 1.32 \mu \mathrm{m}, 5.74 \mu \mathrm{m}$ and $7.5 \mu \mathrm{m}$ and each slit was $0.8 \mu \mathrm{m} \times 0.25 \mu \mathrm{m}$ in size with the smaller size being along the NRA direction [see the inset in Fig. 1(b)]. Double-pinhole apertures had a separation of between $2 \mu \mathrm{m}$ and $15 \mu \mathrm{m}$, whereas the pinhole size varied between $0.34 \mu \mathrm{m}$ and $0.5 \mu \mathrm{m}$. The apertures were mounted on a piezo-positioner stage with a travel range of up to $20 \mathrm{~mm}$. Finally, the interference patterns were recorded by a Spectral Instruments $1100 S$ charge-coupled device $(C C D)$ with $4096 \times 4112$ pixels, each $15 \mu \mathrm{m} \times 15 \mu \mathrm{m}$ in size. The dynamic range of the detector was $10^{4}$. The typical exposure time for one frame of the diffraction patterns was $60 \mathrm{~s}$ for double pinholes and $12 \mathrm{~s}$ for NRA apertures. Each diffraction pattern was an accumulation of three exposures for double pinholes and 30 exposures for NRAs. The detector was positioned $0.8 \mathrm{~m}$ downstream of the sample and a round beamstop, $700 \mu \mathrm{m}$ in diameter, protected the detector from the direct beam.

The transverse coherence and the intensity profile in the vertical direction were measured for different beamline parameters, including the monochromator exit slit size, beamdefining slit size downstream of the undulator, and the photon energy transmitted through the monochromator. The monochromator exit slit width was varied between $40 \mu \mathrm{m}$ and $230 \mu \mathrm{m}$, while the monochromator was tuned to an energy of $400 \mathrm{eV}$ (maximum flux) and the beam-defining slit was opened to $4.7 \mathrm{~mm}$. In this initial stage of beamline commissioning the photon energy resolution was the same for both exit slit openings, resulting in a bandwidth of about $0.5 \mathrm{eV} .^{3}$ This gave

\footnotetext{
${ }^{3}$ At this very early stage of commissioning the grating did not yet deliver the designed resolving power $(E / \Delta E>10000)$.
} 

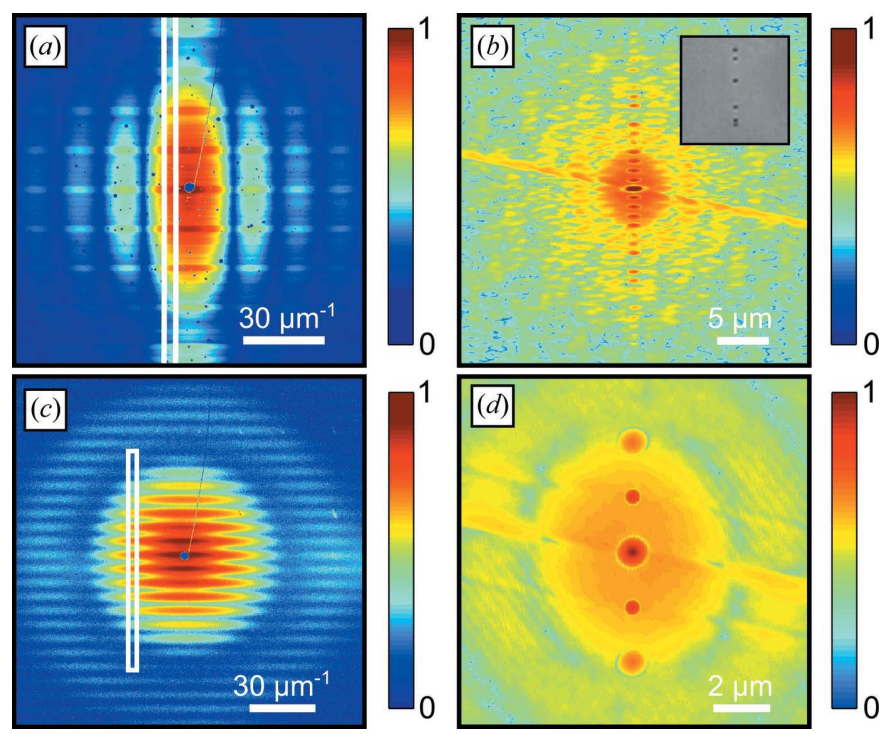

Figure 3

$(a, c)$ Diffraction patterns from NRA apertures with six slits $(a)$ and double pinholes with a separation of $2 \mu \mathrm{m}(c)$ measured at $400 \mathrm{eV}$ photon energy and monochromator exit slits size $D_{\text {es }}=230 \mu \mathrm{m} .(b, d)$ Fourier transforms of the diffraction patterns in $(a, c)$. All figures are displayed on a logarithmic scale. White rectangles in $(a)$ and $(c)$ indicate regions which were used for the analysis. An optical microscope image of the NRA sample is shown in the inset of $(b)$.

us an estimate of the temporal coherence length $l_{\mathrm{t}}=c \tau_{\mathrm{c}}$ that was $l_{\mathrm{t}}=1.6 \mu \mathrm{m}$ in the conditions of our experiment. For the rest of the measurements the exit slit size was fixed to the value $D_{\text {es }}=230 \mu \mathrm{m}$ and the beam-defining slit size $D_{\mathrm{ds}}$ was chosen to be $4.7 \mathrm{~mm}, 1.7 \mathrm{~mm}$ and $0.8 \mathrm{~mm}$. These slits correspond to a relative transmitted flux of $1,0.5$ and 0.25 , respectively. For each beam-defining slit width we measured the transverse coherence at photon energies of $396.5 \mathrm{eV}$, $400 \mathrm{eV}$ and $403 \mathrm{eV}$, which were selected by the monochromator for a fixed undulator gap. At $400 \mathrm{eV}$ the maximum flux was observed, whereas at $396.5 \mathrm{eV}$ and $403 \mathrm{eV}$ the flux was reduced by a factor of two.

Six-slits NRA diffraction patterns were collected for all beamline parameters. Additionally, double-pinhole interferograms were measured for the case of exit slit widths of $40 \mu \mathrm{m}$ and $230 \mu \mathrm{m}$. A sufficient sampling of interference fringes was obtained in all cases with at least 11 pixels per fringe for a $15 \mu \mathrm{m}$ aperture separation.

\section{Results and discussion}

Typical recorded and dark-field-corrected diffraction patterns of six-slits NRA and double pinholes are shown in Figs. 3(a) and 3(c), respectively. Both interferograms were measured at $400 \mathrm{eV}$ photon energy, $4.7 \mathrm{~mm}$ beam-defining slits opening, and monochromator exit slit width $D_{\text {es }}=230 \mu \mathrm{m}$. Peaks corresponding to the aperture interference are clearly visible in the Fourier transforms of both diffraction patterns presented in Figs. 3(b) and 3(d). It is interesting to note that in these Fourier transforms of diffraction patterns the contribution of the higher harmonic radiation at $800 \mathrm{eV}$ photon energy transmitted through the monochromator reveals itself in additional peaks at larger distances visible in Fig. 3(d). Their height is less than $8 \%$ compared with the $400 \mathrm{eV}$ peaks located closer to the central peak. The randomly distributed black spots in Fig. 3(a) are due to contaminations formed at the surface of the detector at the end of the experiment. The streak visible in Figs. $3(b)$ and $3(d)$ is due to a shadow of the beamstop holder visible in Figs. 3(a) and 3(c).

The peak heights $C_{0}$ and $C_{i j}$ for both NRA and doublepinholes diffraction patterns were retrieved from the Fourier transform of a vertical line scan, obtained by averaging 31 pixel-wide regions marked by the white rectangle in Figs. 3(a) and Fig. 3(c). Corresponding line scans of diffraction patterns and their Fourier transforms are shown in Fig. 4. In these regions, high harmonic $800 \mathrm{eV}$ radiation has a minimum
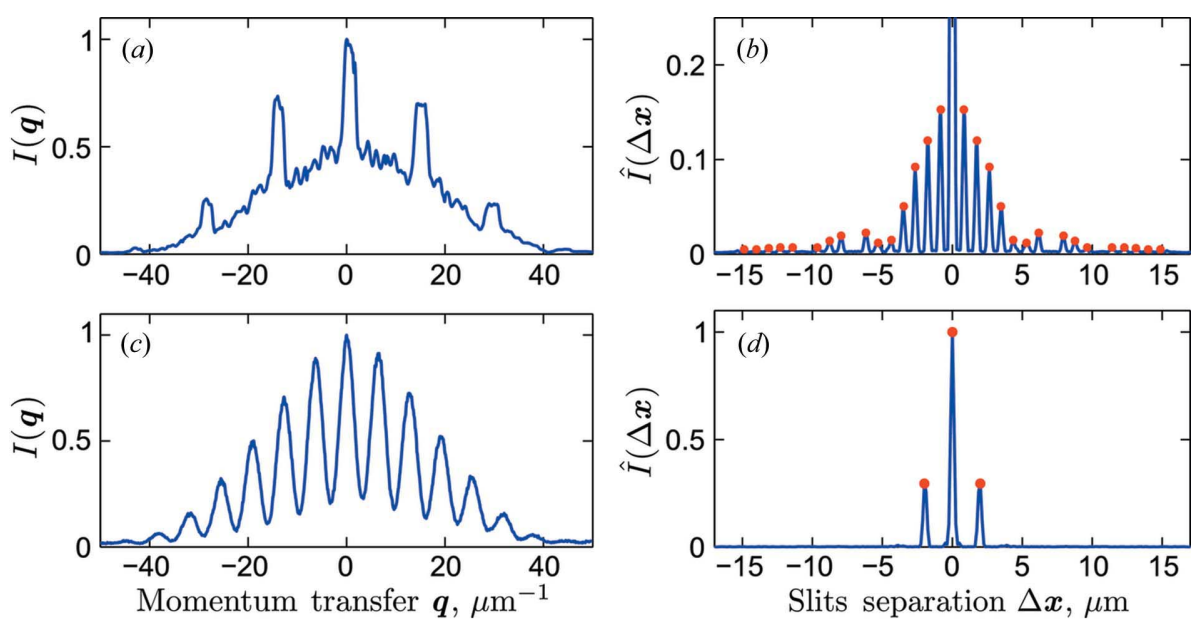

Figure 4

$(a, c)$ Line scans of diffraction patterns $I(q)$ in the vertical direction from the NRA of six slits $(a)$ and double pinholes $(c)$ obtained by averaging the white regions shown in Figs. 3( $a)$ and 3(b), respectively. $(b, d)$ Fourier transforms $\hat{I}(\Delta x)$ of the corresponding line scans from the NRA $(b)$ and double pinholes $(d)$. Red points in $(b, d)$ denote the central and satellite peaks maxima $C_{0}$ and $C_{i j}$. 

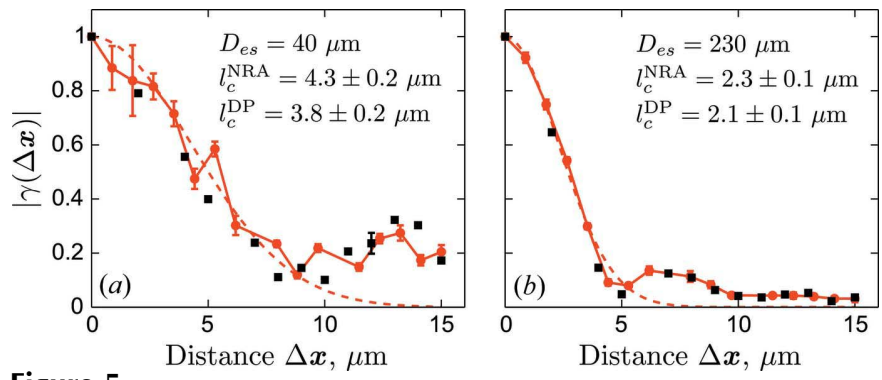

Figure 5

Modulus of the CCF $|\gamma(\Delta x)|$ for $400 \mathrm{eV}$ synchrotron radiation and monochromator exit slit widths of $D_{\mathrm{es}}=40 \mu \mathrm{m}(a)$ and $230 \mu \mathrm{m}(b)$. Red circles, connected with red lines, represent values of the CCF measured by the NRA method and black squares correspond to the values determined by the double pinholes. Gaussian fits for the CCF values obtained with NRAs are shown by dashed red lines.

contribution and temporal coherence effects can be neglected due to small scattering angles. ${ }^{4}$

To determine the intensity distribution in the focus of the beam, the beam profile scans were performed by the double pinholes with a separation of $15 \mu \mathrm{m}$. A position uncertainty of $\pm 1 \mu \mathrm{m}$ was observed during the experiment. Each profile scan was fitted with two Gaussian functions with the same width and $15 \mu \mathrm{m}$ separation. The beam FWHM was determined to be in the range from $10 \mu \mathrm{m}$ to $12 \mu \mathrm{m}$ for the exit slits size $D_{\text {es }}=$ $40 \mu \mathrm{m}$ and in the range from $40 \mu \mathrm{m}$ to $44 \mu \mathrm{m}$ for $D_{\text {es }}=230 \mu \mathrm{m}$. The beam size appeared to be independent of the photon energy and the beam-defining slits width. To define the values of the incident intensities $I_{i}$ at the apertures we assumed that the beam has a Gaussian shape, and is positioned in the center of the NRA aperture with FWHM of $11 \mu \mathrm{m}$ and $42 \mu \mathrm{m}$ for the exit slits $D_{\text {es }}$ of $40 \mu \mathrm{m}$ and $230 \mu \mathrm{m}$, respectively.

Finally, the peak heights $C_{i j}$ and the measured incident intensity profiles $I_{i}$ were used in equation (7) to determine the modulus of the CCF $\left|\gamma_{i j}\right|$. The result from the NRA measurements is presented in Fig. 5 together with the doublepinhole measurements. It is important to note that the results obtained with the NRA of apertures are in excellent agreement with a well established measurement using double pinholes, whereas the measurement time is reduced by an order of magnitude to acquire the same information.

The error bars of the CCF were estimated using a set of incident intensities having FWHM and center of the beam position uncertainty within the error margins given by the intensity measurements. Using equation (7), $\left|\gamma_{i j}\right|$ was calculated from each intensity profile and the uncertainty of $\left|\gamma_{i j}\right|$ was found from the deviation of these values. The errors are comparably large when the beam size is small relative to the NRA size (that was $15 \mu \mathrm{m}$ in our experiment), as in the case of $40 \mu \mathrm{m}$ exit slit opening. ${ }^{5}$ When the FWHM of the focused

\footnotetext{
${ }^{4}$ Our estimates show that the maximum optical path length difference $l$ is about $l=0.4 \mu \mathrm{m}$ in the region chosen for the analysis. This is smaller than the temporal coherence length $l_{\mathrm{t}}=1.6 \mu \mathrm{m}$ and it means that our assumption $\tau<\tau_{\mathrm{c}}$ is well satisfied in this region.

${ }^{5}$ In fact, for the exit slit width of $40 \mu \mathrm{m}$ the incident beam was smaller than the NRA size and we additionally had to find the position of the incident beam relative to the center of the NRA using additional constrains, such as avoiding values of $\left|\gamma_{i j}\right|$ larger than 1, smallest uncertainties and smoothness of the CCF.
}

Table 1

Coherence length $l_{\mathrm{c}}^{\mathrm{NRA}}, l_{\mathrm{c}}^{\mathrm{DP}}$ and normalized degree of coherence $\zeta^{\mathrm{NRA}}$, $\zeta^{\mathrm{DP}}$ measured with NRA and double pinholes (DP) for different exit slit widths of the monochromator $D_{\text {es }}$.

The beam had a FWHM of $11 \pm 1 \mu \mathrm{m}$ in the case of $D_{\mathrm{es}}=40 \mu \mathrm{m}$ and $42 \pm$ $2 \mu \mathrm{m}$ in the case of $D_{\text {es }}=230 \mu \mathrm{m}$. The photon energy was $400 \mathrm{eV}$ and the beam-defining slit was set to $4.7 \mathrm{~mm}$.

\begin{tabular}{lll}
\hline & Exit slit $D_{\text {es }}$ & \\
\cline { 2 - 3 } Parameter & $40 \mu \mathrm{m}$ & $230 \mu \mathrm{m}$ \\
\hline$l_{\mathrm{c}}^{\mathrm{NRA}}(\mu \mathrm{m})$ & $4.3 \pm 0.2$ & $2.4 \pm 0.1$ \\
$l_{\mathrm{c}}^{\text {DP }}(\mu \mathrm{m})$ & $3.8 \pm 0.2$ & $2.1 \pm 0.1$ \\
$\zeta^{\mathrm{NRA}}$ & $0.41 \pm 0.04$ & $0.06 \pm 0.01$ \\
$\zeta^{\mathrm{DP}}$ & $0.38 \pm 0.03$ & $0.06 \pm 0.01$ \\
\hline
\end{tabular}

beam was larger than the total NRA size, as in the case of the $230 \mu \mathrm{m}$ exit slit opening, practically no errors occurred (see Fig. $5 b)$.

To estimate the spatial coherence length $l_{\mathrm{c}}$, the values of the modulus of the CCF $|\gamma(\Delta x)|$ were approximated by a Gaussian $\exp \left[-\Delta x^{2} /\left(2 l_{\mathrm{c}}^{2}\right)\right]$. Results of our evaluation for the NRA as well as for the double pinholes are presented in Table 1. The values of the global degree of coherence $\zeta$ [see equation (3)] are also given in the same table. These results demonstrate an excellent agreement between both methods.

Finally, we present spatial coherence measurements performed with NRA apertures for the cases of $396.5 \mathrm{eV}$, $400 \mathrm{eV}$ and $403 \mathrm{eV}$ beam energies as well as $4.7 \mathrm{~mm}, 1.7 \mathrm{~mm}$ and $0.8 \mathrm{~mm}$ beam-defining slit width. In all cases the monochromator exit slit width was set to $D_{\mathrm{es}}=230 \mu \mathrm{m}$. The results of these measurements are presented in Fig. 6. The transverse coherence length $l_{\mathrm{c}}$ for all curves was determined by the Gaussian fit, as before, and the result is summarized in Table 2.

As expected, the coherence length $l_{\mathrm{c}}$ is inversely proportional to the beam-defining slit width. The slit widths were chosen such that the transmitted intensity drops by a factor of two in each step. Interestingly, the normalized degree of coherence $\zeta$ increases by about a factor of two in each step. This means that in the range accessed in our experiment the beam-defining slit cuts out the coherent part of the beam and almost no coherent flux is lost. For the blue-shifted beam $(403 \mathrm{eV})$ this relation does not hold due to a high value of the normalized degree of coherence (Singer \& Vartanyants, 2011).

We also observed a variation of the transverse coherence length as a function of the monochromator tuning energy. The energies were chosen such that the maximum flux at $400 \mathrm{eV}$ was reduced by a factor of two for both energies $403 \mathrm{eV}$ and $396.5 \mathrm{eV}$. For the largest beam-defining slit $(4.7 \mathrm{~mm})$ the coherence length increases by $25 \%$ when the monochromator is detuned to $403 \mathrm{eV}$. However, unlike in the case of the beamdefining slit the coherent flux is reduced. Interestingly, the functional form appears to be closer to a Gaussian function for the blue-shifted radiation. We want to note here that it is the power of the method here that allows these fine details of the coherence function to be recorded. For an energy of $396.5 \mathrm{eV}$ 

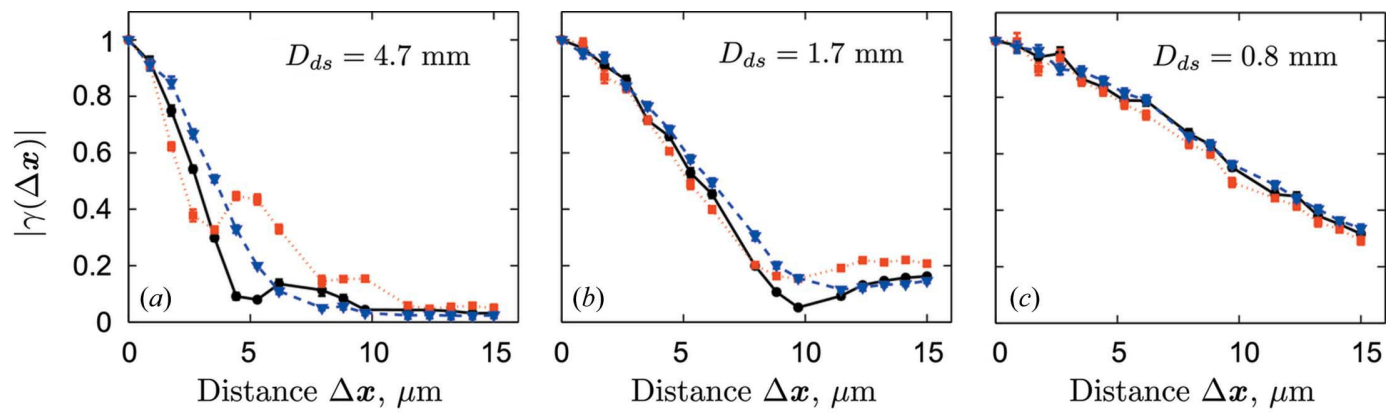

Figure 6

Modulus of the CCF $|\gamma(\Delta x)|$ measured for an exit slit width of $D_{\mathrm{es}}=230 \mu \mathrm{m}$ and beam-defining slit widths of $D_{\mathrm{ds}}=4.7 \mathrm{~mm}(a), 1.7 \mathrm{~mm}(b)$ and $0.8 \mathrm{~mm}$ (c). Measurements performed at $396.5 \mathrm{eV}$ (red squares), $400 \mathrm{eV}$ (blue triangles) and $403 \mathrm{eV}$ (black circles) are presented.

Table 2

Coherence length $l_{\mathrm{c}}$ and normalized degree of coherence $\zeta$ for different photon energies and beam-defining slit size $D_{\mathrm{ds}}$ obtained as a result of the analysis of data presented in Fig. 6.

The monochromator exit slits were fixed to $D_{\mathrm{es}}=230 \mu \mathrm{m}$.

\begin{tabular}{lllll}
\hline & & \multicolumn{3}{l}{ Beam-defining slit $D_{\mathrm{ds}}$} \\
\cline { 3 - 5 } Energy $(\mathrm{eV})$ & Parameter & $4.7 \mathrm{~mm}$ & $1.7 \mathrm{~mm}$ & $0.8 \mathrm{~mm}$ \\
\hline \multirow{2}{*}{396.5} & $l_{\mathrm{c}}(\mu \mathrm{m})$ & $1.9 \pm 0.1$ & $4.4 \pm 0.1$ & $8.8 \pm 0.3$ \\
& $\zeta$ & $0.05 \pm 0.01$ & $0.12 \pm 0.01$ & $0.24 \pm 0.01$ \\
400 & $l_{\mathrm{c}}(\mu \mathrm{m})$ & $2.4 \pm 0.1$ & $4.6 \pm 0.1$ & $9.2 \pm 0.2$ \\
& $\zeta$ & $0.06 \pm 0.01$ & $0.13 \pm 0.01$ & $0.25 \pm 0.01$ \\
403 & $l_{\mathrm{c}}(\mu \mathrm{m})$ & $3.0 \pm 0.1$ & $5.1 \pm 0.1$ & $9.4 \pm 0.2$ \\
& $\zeta$ & $0.08 \pm 0.01$ & $0.14 \pm 0.01$ & $0.25 \pm 0.01$ \\
\hline
\end{tabular}

the transverse coherence is worse. ${ }^{6}$ The energy dependence of the transverse coherence is significantly smaller (about $5 \%$ ) for the smallest beam-defining slit.

\section{Conclusions and outlook}

In conclusion, a non-redundant array of apertures provides a fast and effective way to determine the transverse coherence function of the undulator radiation in one direction. We showed that in this method a single diffraction pattern is sufficient to find the degree of coherence of X-ray radiation at several relative distances simultaneously. The results obtained with NRA apertures concord well with the values determined using Young's double pinholes, whereas the measurement time was reduced significantly. The dependence of spatial coherence on different parameters was explored. The transverse coherence length shows an inverse proportional dependence on both the width of the beam-defining slit and the exit slit. We also observed that the spatial coherence improves if the monochromator is offset to larger energies (blue-shifted) and it is decreased for smaller energies (redshifted). We anticipate that the method presented here will be a useful procedure for XUV and soft X-ray beam calibration prior to any experiment utilizing spatial coherence. A natural extension of this method will be to obtain transverse coher-

\footnotetext{
${ }^{6}$ This effect could be due to the fact that on the higher-energy side of the undulator peak the collimation of the undulator cone is better than towards the lower-energy side where at even lower energies an intensity minimum is observed on-axis, resulting in a non-optimal beam path.
}

ence properties of the beam in two dimensions simultaneously. This can be done, for example, by designing two-dimensional NRA apertures as proposed by González \& Mejía (2011). We also foresee that in future the NRA method could become a viable tool for single-pulse coherence characterization measurements at free-electron lasers (Singer et al., 2008, 2012; Vartanyants et al., 2010, 2011).

We acknowledge fruitful discussions and support of the project by E. Weckert, help with the manufacturing of the NRA apertures by D. Attwood and Y. Liu, and help with the design of the NRA apertures by A. Mancuso. We are thankful to F. Scholz, I. Shevchuk and J. Seltmann for setting up the experiment at $\mathrm{P} 04$ beamline. Part of this work was supported by $\mathrm{BMBF}$ proposal $05 \mathrm{~K} 10 \mathrm{CHG}$ 'Coherent Diffraction Imaging and Scattering of Ultrashort Coherent Pulses with Matter' in the framework of the German-Russian collaboration 'Development and Use of Accelerator-Based Photon Sources' and the Virtual Institute VH-VI-403 of the Helmholtz Association. Additional support by BMBF under 05K10GU4, by the DFG within SFB925 and from the Hamburg Centre for Ultrafast Imaging is greatly acknowledged.

\section{References}

Alaimo, M. D., Potenza, M. A. C., Manfredda, M., Geloni, G., Sztucki, M., Narayanan, T. \& Giglio, M. (2009). Phys. Rev. Lett. 103, 194805. Attwood, D. (1999). Soft X-rays and Extreme Ultraviolet Radiation. Cambridge University Press.

Bartels, R. A., Paul, A., Green, H., Kapteyn, H. C., Murnane, M. M., Backus, S., Christov, I. P., Liu, Y., Attwood, D. \& Jacobsen, C. (2002). Science, 297, 376-378.

Chang, C., Naulleau, P., Anderson, E. \& Attwood, D. (2000). Opt. Commun. 182, 25-34.

Chapman, H. N. \& Nugent, K. A. (2010). Nat. Photon. 4, 833-839.

Dollas, A., Rankin, W. T. \& McCracken, D. (1998). IEEE Trans. Inf. Theory, 44, 379-382.

Eisebitt, S., Lüning, J., Schlotter, W. F., Lörgen, M., Hellwig, O., Eberhardt, W. \& Stöhr, J. (2004). Nature (London), 432, 885-888. Fienup, J. R. (1982). Appl. Opt. 21, 2758-2769.

Gluskin, E., Alp, E. E., McNulty, I., Sturhahn, W. \& Sutter, J. (1999). J. Synchrotron Rad. 6, 1065-1066.

González, A. I. \& Mejía, Y. (2011). J. Opt. Soc. Am. A, 28, 1107-1113. Goodman, J. W. (1985). Statistical Optics. New York: Wiley.

Goodman, J. W. (2007). Speckle Phenomena in Optics. Greenwood Village: Roberts. 
Grübel, G. \& Zontone, F. (2004). J. Alloys Compd. 362, 3-11.

Lam, A. W. \& Sarwate, D. V. (1988). IEEE Trans. Commun. 36, 380 382.

Lin, J. J. A., Paterson, D., Peele, A. G., McMahon, P. J., Chantler, C. T., Nugent, K. A., Lai, B., Moldovan, N., Cai, Z., Mancini, D. C. \& McNulty, I. (2003). Phys. Rev. Lett. 90, 074801.

Mancuso, A. P. et al. (2010). New J. Phys. 12, 035003.

Mejía, Y. \& González, A. I. (2007). Opt. Commun. 273, 428-434.

Paterson, D., Allman, B. E., McMahon, P. J., Lin, J., Moldovan, N., Nugent, K. A., McNulty, I., Chantler, C. T., Retsch, C. C., Irving, T. H. K. \& Mancini, D. C. (2001). Opt. Commun. 195, 79-84.

Pfeiffer, F., Bunk, O., Schulze-Briese, C., Diaz, A., Weitkamp, T., David, C., van der Veen, J. F., Vartanyants, I. \& Robinson, I. K. (2005). Phys. Rev. Lett. 94, 164801.

Raymer, M. G., Beck, M. \& McAlister, D. (1994). Phys. Rev. Lett. 72 , 1137-1140.

Saldin, E. L., Schneidmiller, E. A. \& Yurkov, M. V. (2008). Opt. Commun. 281, 1179-1188.

Singer, A. et al. (2012). Opt. Express, 20, 17480-17495.

Singer, A., Lorenz, U., Sorgenfrei, F., Gerasimova, N., Gulden, J., Yefanov, O. M., Kurta, R. P., Shabalin, A., Dronyak, R., Treusch, R., Kocharyan, V., Weckert, E., Wurth, W. \& Vartanyants, I. A. (2013). Phys. Rev. Lett. 111, 034802.

Singer, A. \& Vartanyants, I. A. (2011). Proc. SPIE, 8141, 814106.
Singer, A., Vartanyants, I. A., Kuhlmann, M., Duesterer, S., Treusch, R. \& Feldhaus, J. (2008). Phys. Rev. Lett. 101, 254801.

Stickler, D., Frömter, R., Stillrich, H., Menk, C., Tieg, C., StreitNierobisch, S., Sprung, M., Gutt, C., Stadler, L.-M., Leupold, O., Grübel, G. \& Oepen, H. P. (2010). Appl. Phys. Lett. 96, 042501.

Tran, C. Q., Williams, G. J., Roberts, A., Flewett, S., Peele, A. G., Paterson, D., de Jonge, M. D. \& Nugent, K. A. (2007). Phys. Rev. Lett. 98, 224801.

Vartanyants, I. A., Mancuso, A. P., Singer, A., Yefanov, O. M. \& Gulden, J. (2010). J. Phys. B: At. Mol. Opt. Phys. 43, 194016.

Vartanyants, I. A. \& Singer, A. (2010). New J. Phys. 12, 035004.

Vartanyants, I. A. et al. (2011). Phys. Rev. Lett. 107, 144801.

Viefhaus, J., Scholz, F., Deinert, S., Glaser, L., Ilchen, M., Seltmann, J., Walter, P. \& Siewert, F. (2013). Nucl. Instrum. Methods Phys. Res. A, 710, 151-154.

Whitehead, L. W., Williams, G. J., Quiney, H. M., Vine, D. J., Dilanian, R. A., Flewett, S., Nugent, K. A., Peele, A. G., Balaur, E. \& McNulty, I. (2009). Phys. Rev. Lett. 103, 243902.

Wikipedia (2014). Golomb ruler. Wikipedia, the Free Encyclopedia. [Online; accessed 19 January 2014.]

Winick, H. (1980). Synchrotron Radiation Research, edited by H. Winick and S. Doniach. New York/London: Plenum.

Yabashi, M., Tamasaku, K. \& Ishikawa, T. (2001). Phys. Rev. Lett. 87, 140801. 